\title{
Thunder-fire moxibustion for cervical spondylotic radiculopathy: Study protocol for a randomized controlled trial
}

\author{
Yunxuan Huang ${ }^{1}$, Jiabi Zhang ${ }^{2}$, Buhui Xiong ${ }^{2}$, Ruina Huang ${ }^{3}$, Wenjing Zhao ${ }^{4}$, Mengxue Zhou ${ }^{5}$, Qi Chen ${ }^{2}$,
} Danghan $\mathrm{Xu}^{1^{*}}$ (1) and Xinghua Chen ${ }^{1^{*}}$ (D)

\begin{abstract}
Background: Thunder-fire moxibustion originated in China and contains traditional Chinese medicine. It can produce strong firepower, infrared thermal radiation, and medicinal effects when burning on the acupoints. Thunder-fire moxibustion is commonly used in patients with neck pain, but its efficacy has rarely been systematically demonstrated. We designed a randomized trial of thunder-fire moxibustion on cervical spondylotic radiculopathy (CSR) to investigate whether it is more effective than ibuprofen sustained-release capsules.

Methods: One hundred patients will be recruited and randomly divided into thunder-fire moxibustion and ibuprofen groups. The intervention consists of ten treatments and will last for 2 weeks. The Yasuhisa Tanaka 20 Score Scale is used as the primary outcome measure. It contains a combination of the self-conscious symptom in patients, objective clinical evaluation from doctors, and social evaluation (the ability to work and live). The objective and comprehensive evaluation of CSR patients before and after treatment is particularly needed. The Short-Form McGill Pain Questionnaire-2 (SF-MPQ-2), Neck Disability Index score scale (NDI), and the Quality of Life Assessment (SF-36) are applied as secondary outcome measures. The assessment will take place at the baseline and the first and second weekends of treatment. If an adverse event (AEs) occurs, it will be reported.
\end{abstract}

Discussion: The aim of this trial is to determine whether thunder-fire moxibustion is more effective than ibuprofen in the treatment of patients with CSR.

Trial registration: Chinese Clinical Trial Registry (http://www.chictr.org.cn), ChiCTR1800018820. Registered on 11 October 2018

Keywords: Cervical spondylotic radiculopathy, Thunder-fire moxibustion, Ibuprofen, RCT

\section{Background}

Cervical spondylotic radiculopathy (CSR) is characterized by the dysfunction of a cervical spinal nerve, the roots of the nerve, or both $[1,2]$. Patients with cervical spondylotic radiculopathy suffer deeply from numbness and pain of the arm and neck. According to the Global Burden of Disease Study (2013) [3], among 301 chronic and acute injuries and illnesses in 188 countries, neck pain was one of the top ten causes of disability of years. Furthermore, neck

\footnotetext{
* Correspondence: xudanghan@126.com; chenxinghua@gzucm.edu.cn ${ }^{1}$ The First Affiliated Hospital of Guangzhou University of Chinese Medicine, No.12 Ji Chang Road, Baiyun District, Guangzhou 510405, China Full list of author information is available at the end of the article
}

activities are restricted. CSR, as a common type of cervical spondylosis (CS), accounts for about 60 to $70 \%$ [4].

Currently, the approved CSR treatment strategies contain surgical and non-surgical treatment, including traction, drugs, functional exercise, and physical therapy, etc. $[5,6]$.

In practice, analgesics are standard primary treatments for CSR unless there is evidence of spinal cord disease or apparent inability to move. Nonsteroidal antiinflammatory drugs (NSAIDs) could relieve pain as firstline agents in acute settings [7]. The efficacy of ibuprofen in the treatment of cervical nerve root pain has also been reported [8], but it can only relieve pain and has no effect on the other symptoms of CSR.

(c) The Author(s). 2020 Open Access This article is distributed under the terms of the Creative Commons Attribution 4.0 International License (http://creativecommons.org/licenses/by/4.0/), which permits unrestricted use, distribution, and reproduction in any medium, provided you give appropriate credit to the original author(s) and the source, provide a link to the Creative Commons license, and indicate if changes were made. The Creative Commons Public Domain Dedication waiver (http://creativecommons.org/publicdomain/zero/1.0/) applies to the data made available in this article, unless otherwise stated. 
In the treatment of CS neck pain, complementary therapies such as acupuncture, moxibustion, and massage have been widely accepted [9]. Thunder-fire moxibustion, which contains refined moxa as well as agarwood, frankincense, costus root, and other traditional Chinese medicines, is often used in China to warm the meridian to relieve pain [10]. However, the effect of thunder-fire moxibustion on CSR remains uncertain because of poor study design and small sample sizes in previous clinical trials [10-13].

In this study, the target is to investigate and compare the efficacy of thunder-fire moxibustion with oral application of ibuprofen sustained-release capsules in patients with CSR suffering from pain, numbness, and dysfunction.

\section{Methods}

Trial design

We have designed a single-center randomized controlled trial (RCT) to compare thunder-fire moxibustion with ibuprofen in CSR patients. Participants will receive ten treatments within 2 weeks.

One hundred patients with CSR will be recruited and randomly assigned to either the thunder-fire moxibustion group or an ibuprofen group by a 1:1 ratio (Fig. 1). Our study is based on the rule of common clinical trials (Declaration of Helsinki). The SPIRIT checklist is given in Additional file 1.

\section{Inclusion criteria}

Participants will be enrolled with the following criteria in this study: (1) men or women aged from 18 to 65 years, (2) the main signs and symptoms are numbness and pain along spinal nerve roots, (3) intervertebral foramen extrusion and/or brachial plexus pull tests is/are positive,(4) the clinical manifestations and imaging are in compliance with the clinical syndromes, and (5) participants who can understand the scales, i.e., those used to measure trial outcomes.

\section{Exclusion criteria}

Subjects will be excluded if they meet one of the following criteria: (1) tennis elbow, tendonitis of biceps brachii, periarthritis of the shoulder, acute spinal cord injury, or syndrome of cubital tunnel, carpal tunnel, thoracic outlet; (2) symptoms of cervical vertigo, acute spinal cord inflammation, and abnormal changes on transcranial Doppler (TCD); (3) acupuncture treatment or medication for the disease during the past 1 month; (4)acute or chronic infection, trauma, fever or allergy during the previous 2 weeks; (5) any other situations, which includes taking anticoagulant, clotting disorders, active skin infection; (6) severe primary disease or infectious diseases including kidney, heart or liver disease, hematopoietic system, endocrine system disease, or pregnancy or breastfeeding; (7) a severe psychological disorder or psychiatric condition associated with dementia and severe neurosis and inability to communicate or take care of oneself; (8) patients who are afraid of moxibustion.

\section{Recruitment}

We will recruit participants by advertising on bulletin boards, located at the Department of Orthopedics, the Department of Acupuncture and Characteristic Chinese Medicine, and the Department of Rehabilitation Nursing Center at the First Affiliated Hospital of Guangzhou University of Chinese Medicine. Recruitment staff working in these departments will be in charge of the recruitment and registration of the participants who meet the

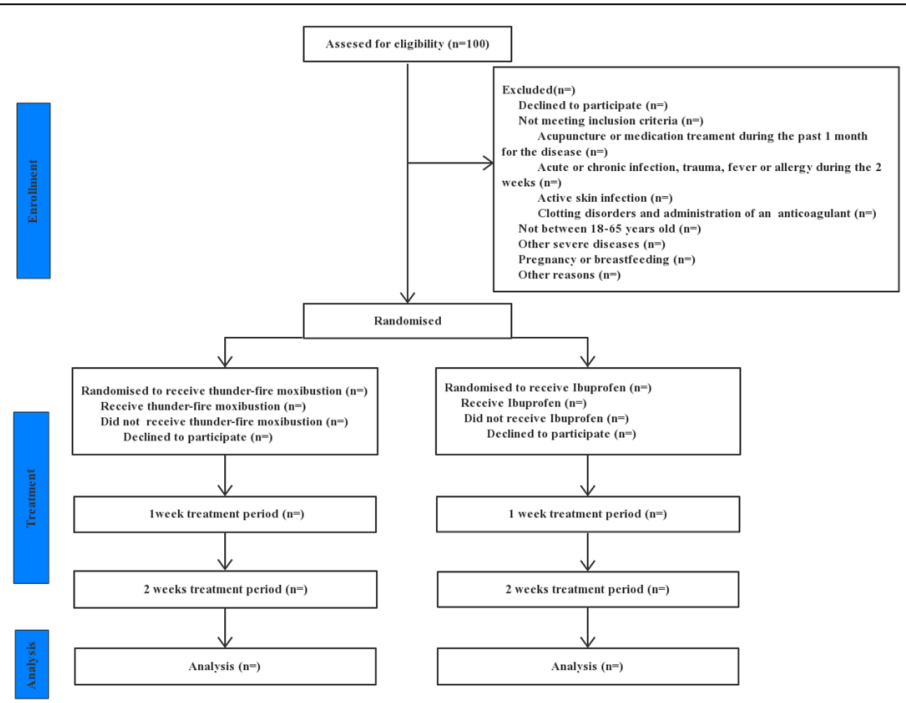

Fig. 1 Trial flow chart 
inclusion criteria. The details about the participants will be maintained by the Data Monitoring Committee (DMC) and will never be revealed to any other individual or organization irrelevant to this study.

\section{Randomization and allocation concealment}

A randomized block design is used for the study. Patients with CSR will be randomly assigned to the group of thunder-fire moxibustion or ibuprofen in a 1:1 ratio. The random list will be generated by an independent statistician by a block size of 6 using SAS 9.1 software. The random numbers list generated will be concealed using opaque and sealed envelopes with an independent custodian.

\section{Blinding}

This is an open-label study. However, researchers consisting of outcome measurers and statisticians in the trial should be blinded to reduce the impact of subjective impressions.

\section{Interventions}

Moxibustion staff members who have more than 1-yearexperience in thunder-fire moxibustion will be called for this trial and retrained for standard operation before the trial. The moxa-cigars for the moxibustion are produced by the Traditional Medicine Research Institute of Zhao's thunder-fire moxibustion. Each moxa-cigar is $10 \times 3 \mathrm{~cm}$ and weighs $25 \mathrm{~g}$. The treatment site is the local neck, using acupoint selections BL10 (Tianzhu), BL11 (Dazhu), and EX-HN15 (Jingbailao). The subject will be in a relaxed prone position. One moxa-cigar is placed in a 1hole moxibustion box, and the tops of the moxa-cigars are lit. The moxibustion box is then placed on the treatment site, with the fire head $2-3 \mathrm{~cm}$ from the skin. Moxibustion is performed on the local neck area for a treatment of $30 \mathrm{~min}$. The moxibustion box and the treatment area are covered with a thick treatment towel to maintain temperature and to control smoke (Figs. 2 and 3). Treatments will be given 5 days per week for 2 weeks. The treatment using ibuprofen from SK\&F $(0.3$ g per tablet) will also last 2 weeks, with two tablets per day taken orally.

\section{Outcome measurements}

All the outcome measurements will take place at the baseline (before treatment) and the first and second weekend during treatment (Fig. 4).

\section{Primary outcome measurement}

Yasuhisa Tanaka 20 Score Scale The Yasuhisa Tanaka 20 Score Scale [14-16] has proven its efficiency to assess cervical spondylotic radiculopathy. It consists of nine items, which includes the severity of neck pain, stupefaction, and the pain from upper limbs or fingers. Signs include intervertebral orifice extrusion test, sensation,

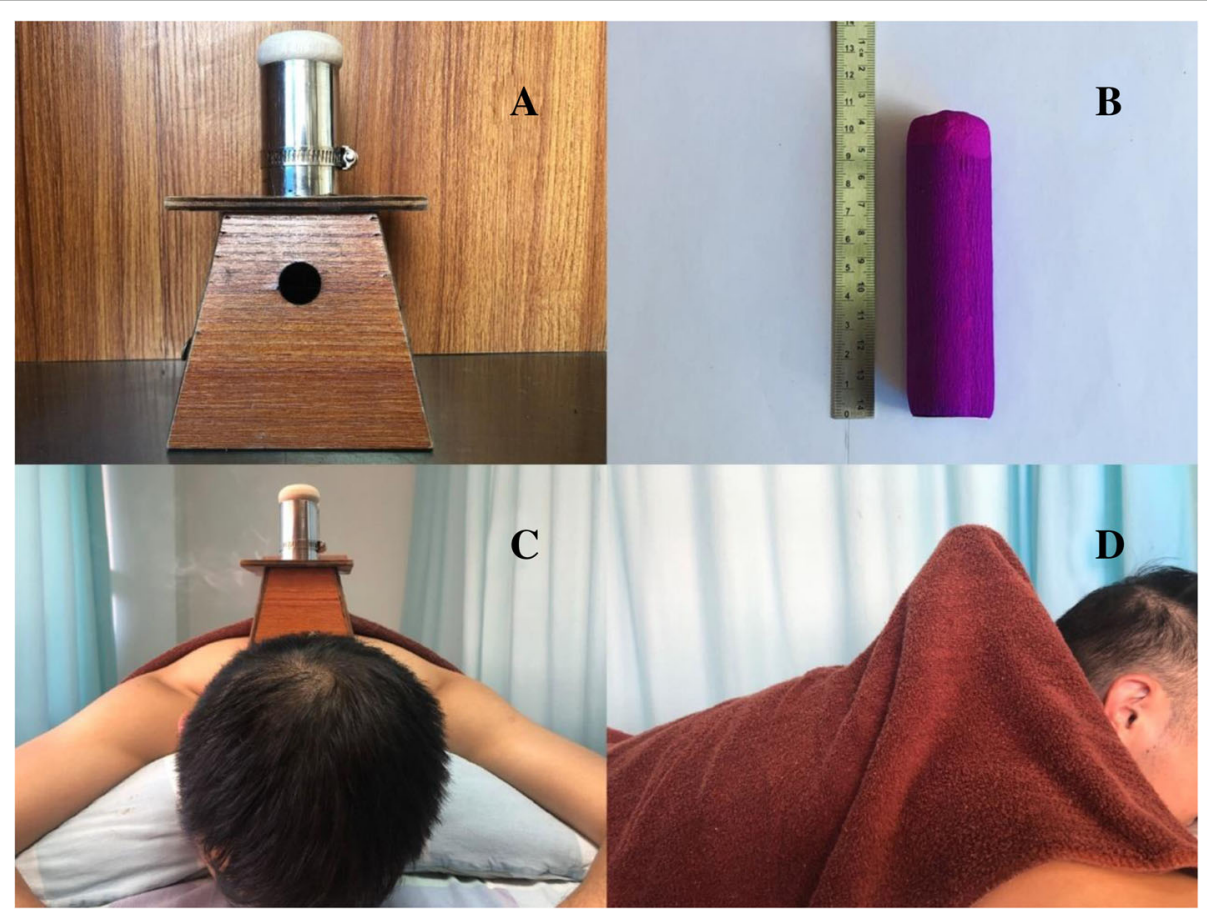

Fig. 2 Thunder-fire moxibustion. Picture $\mathbf{A}$ is the 1-hole moxibustion box. Picture $\mathbf{B}$ is a moxa-cigar. Picture $\mathbf{C}$ shows that the moxibustion box is placed on the treatment site. Picture $\mathbf{D}$ shows that the moxibustion box and the treatment area are covered with a thick treatment towel 


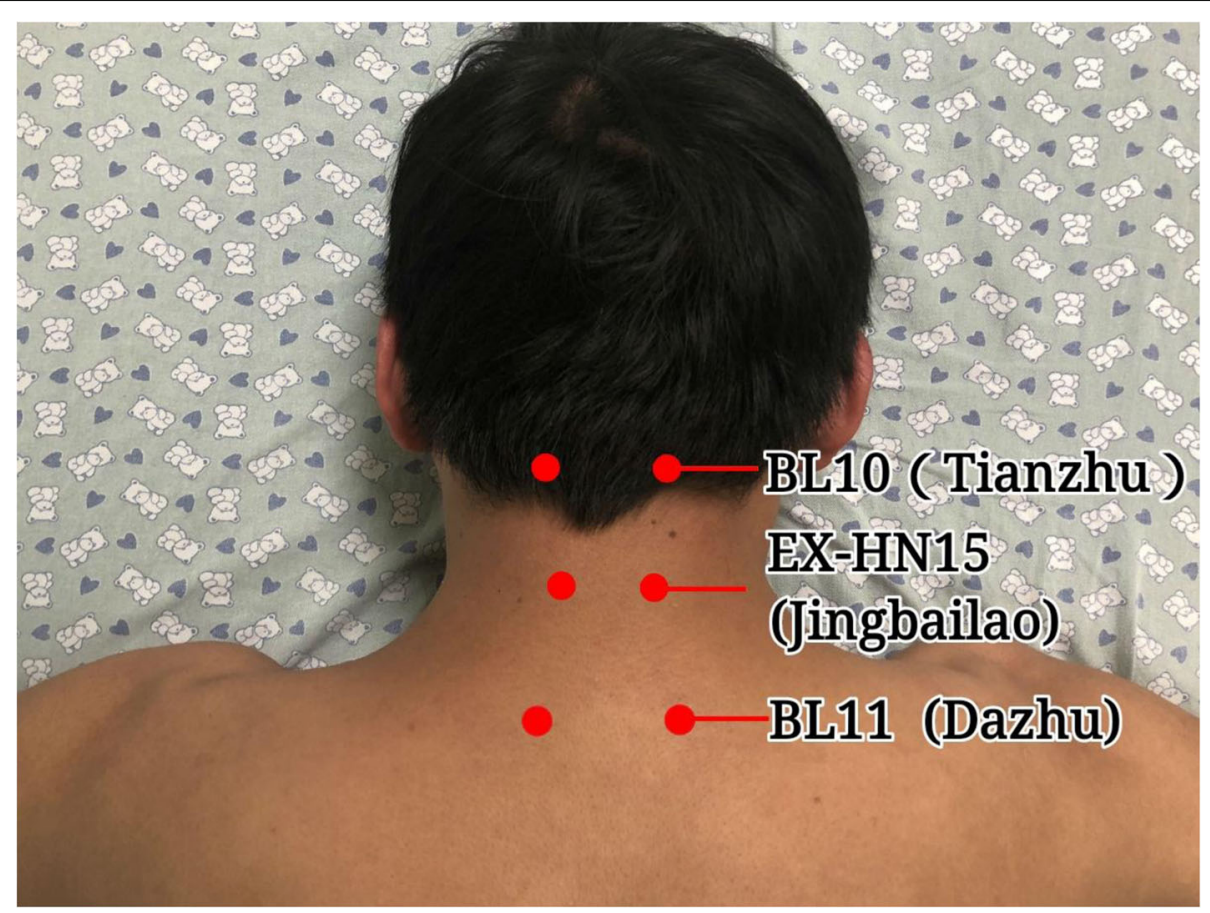

Fig. 3 Location of acupoints

muscle strength, tendon reflex, and function of the hand. Working and living ability is also included. Low scores indicate worse pain and disability.

\section{Secondary outcome measurement}

\section{Short-Form McGill Pain Questionnaire-2 (SF-MPQ-2)}

[17] There are four parts in this questionnaire: (1) continuous pain such as cramping ache, aching pain, tender ache, gnawing ache, and heavy ache; (2) intermittent soreness, for instance, shooting hurt, sharp hurt, splitting hurt, stabbing hurt, and electric-shock hurt; (3) neuropathic pain including hot-burning ache, cold-freezing ache, "pins and needles" or tingling, ache caused by light touch, itching, and numbness; (4) emotional overtired, unwholesome, scared, and excruciating scale. Four different scores were added to get the final scores. A higher score represents more severe pain.

Neck Disability Index Score Scale (NDI) [18, 19] Neckspecific functional disability will be measured by the original ten-item Neck Disability Index (NDI). The NDI covers ten dimensions including entertainment, sleep, driving, headache, reading, personal care, work, lifting, concentration, and pain intensity. One dimension will be assessed on each item measured by a 6-point scale from 0 (no disability) to 5 (complete disability). The scores of each item will be added and multiplied by 2 to get the final total score (out of 100).

Quality of Life Assessment (SF-36) [20] The 36-Item Short-Form Health Survey (SF-36) is a concise health measurement scale developed by the Boston Institute of Health. It has eight dimensions and 36 clauses, including the dimensions physical role limitation (RP), physical functioning $(\mathrm{PF})$, general health $(\mathrm{GH})$, bodily pain $(\mathrm{BP})$, vitality $(\mathrm{V})$, emotional role limitation (RE), mental health $(\mathrm{MH})$, and social functioning (SF). Questions and answers will be transformed into a point scale ranging from 0 to 100 . High marks mean severe damage due to cervical spondylotic radiculopathy.

\section{Sample-size calculation}

This study aims to estimate the exact effect of thunderfire moxibustion compared to ibuprofen. The sample size has been estimated based on the results of a previous study that had shown an extract of acupuncture and moxibustion eases CSR [10]. The Yasuhisa Tanaka 20 Score Scale will be used as the primary outcome measure to assess the analgesic effect of thunder-fire moxibustion in this trial. Results from a previous study showed that the mean changes in the Yasuhisa Tanaka 20 Score Scale were $8.27 \pm 2.48$ (acupuncture and moxibustion group) and $6.77 \pm 2.58$ (control group) [10] SPSS14 software was used to calculate the sample size. 


\begin{tabular}{|c|c|c|c|c|}
\hline \multirow[b]{3}{*}{ TIMEPOINT } & \multicolumn{4}{|c|}{ STUDY SCHEDULE } \\
\hline & \multirow{2}{*}{$\frac{\text { Screening }}{\text { Week-1 }}$} & \multirow{2}{*}{$\begin{array}{c}\text { Baseline } \\
\text { Weeko }\end{array}$} & \multicolumn{2}{|c|}{ Intervention (Week1-2) } \\
\hline & & & Week1 & Week2 \\
\hline \multicolumn{5}{|c|}{ ENROLMENT } \\
\hline Eligibility screen & $X$ & & & \\
\hline Informed consent & $X$ & & & \\
\hline Allocation & & $X$ & & \\
\hline \multicolumn{5}{|c|}{ INTERVENTIONS } \\
\hline $\begin{array}{l}\text { Thunder-fire } \\
\text { moxibustion group }\end{array}$ & & & $\mathrm{X}$ & $\mathrm{X}$ \\
\hline Ibuprofen group & & & $X$ & $X$ \\
\hline \multicolumn{5}{|c|}{ OUTCOMES } \\
\hline $\begin{array}{c}\text { Yasuhisa Tanaka } 20 \\
\text { Score Scale }\end{array}$ & & $\mathrm{X}$ & $\mathrm{X}$ & $\mathrm{X}$ \\
\hline SF-MPQ-2, & & $X$ & $X$ & $X$ \\
\hline NDI & & $\mathrm{X}$ & $\mathrm{X}$ & $x$ \\
\hline SF-36 & & $X$ & $X$ & $X$ \\
\hline \multicolumn{5}{|c|}{ MONITOR } \\
\hline Adverse events & & & $\mathrm{X}$ & $X$ \\
\hline Patient's compliance & & & $X$ & $X$ \\
\hline Trial Continuity & & & $X$ & $x$ \\
\hline
\end{tabular}

Fig. 4 Study schedule. Week 1: the 5th treatment during the first week. Week 2: the 10th treatment during the second week. All outcomes take place at baseline, the first weekend, and the second weekend during treatment

The confidence of the trial sample size was $90 \%$ and the significance level was 0.05 . Results showed that clinically significant differences would be detected using a minimum sample size of 46 individuals in each group. The maximum permissible drop-out rate was $10 \%$ and 100 subjects (50 per group) were recruited.

\section{Statistical analysis}

To eliminate artificial error, two statisticians will be involved to independently run statistical analysis via SPSS software (version 24.0) and R statistical package (version 3.5.0).

If the continuous variables can meet a normal distribution or $\mathrm{T}$ distribution, the data between the two groups will be compared by Student's $t$ test. Otherwise, we will use the Mann-Whitney test or Wilcoxon test. For categorical data, the Fisher's exact or the Chi-square test will be adopted. It is statistically significant when the $p$ value is under 0.05 . We will select the intention-to-treat principle to perform this statistical analysis. Thus, multiple imputations will be used to address the missing data. We will use two different methods: intention-totreat and per-protocol, for sensitivity analysis. Moreover, we will establish a multiple regression model to control the covariates. We can calculate the independent effect of each variable on the outcome via this model, which will provide data for our further trials.

\section{Safety and adverse events}

The group of thunder-fire moxibustion may encounter adverse events including xerostomia, constipation, skin burn, bleeding tendency, dizziness, and fainting [21-23]. If the above situation occurs, the thunder-fire moxibustion treatment should be stopped immediately, and the participants should drink warm water and have a rest. Adverse events caused by oral ibuprofen mainly include tinnitus, dizziness, drowsiness, skin rash, nausea, vomiting, abdominal distension, itching, dyspepsia, and 
blurred vision. Once these adverse reactions occur, participants must stop the ibuprofen and select symptomatic treatment if necessary. These AEs will be subcategorized by severity: mild, moderate, and severe adverse events (mild adverse events = adverse events are transient and tolerable; moderate adverse events =adverse events will cause discomfort and interfere with the subject's normal life; severe adverse events = serious impact on the participants' physical health and even lead to the risk of life). The record form will be filled in if adverse events occur during the treatment period including the time, duration, performance, measures to be taken, and the outcome.

\section{Data management and monitoring}

A case report form (CRF) will be used in data collection. Data information on demographics and assessment after each treatment of every participant will be recorded completely by the data monitoring committee. The cause of patient drop-out should be clarified in the CRF for all shedding cases. At the end of the study, the investigator will submit the case report form to the data management committee for all patients enrolled in the trial. Continuity of the trial will be assessed if more than $25 \%$ of the patients discontinue intervention due to moderate or severe adverse events.

The data monitoring committee is independently chaired by the Statistics Teaching and Research Office of Guangzhou University of Chinese Medicine and claims to have no conflict of interest. The South China Research Center for Acupuncture and Moxibustion will act as an independent committee to monitor the progress and provide advice if necessary. The Ethics Committee of the First Affiliated Hospital of Guangzhou University of Chinese Medicine will take part in endpoint adjudication. All staff members will be included in the author's contribution.

The Project Management Group will meet every week to review trial conduct. The Trial Steering Group will meet every month, and the independent Data Monitoring and Ethics Committee will meet every 6 months to review conduct throughout the trial period.

\section{Discussion}

The pathogenesis factors of CSR include cervical degeneration, trauma, strain, cervical dysplasia, inflammation, wind, cold and wet environment, etc. Modern medicine holds that CSR originates from the secondary inflammatory injury caused by nerve root stimulation and hyperplasia due to cervical disc herniation or joint hypertrophy, resulting in arm and shoulder numbness and pain [1]. Modern medicine mostly adopts drug treatment, rehabilitation treatment, and surgical treatment. Nevertheless, traditional Chinese medicine has a proven remarkable effect on the treatment of CSR with acupuncture, massage, and moxibustion.

Since ancient times, moxibustion therapy has been widely used in the prevention and treatment of a variety of systemic diseases, including digestion, exercise, respiration, cardiovascular, and urinary systems, etc. Furthermore, in clinical practice, moxibustion treatment of cervical spondylosis has achieved a certain effect. However, according to the literature, moxibustion is usually accompanied by other therapies, such as acupuncture and massage, for the treatment of cervical spondylosis. Design of these combined treatments may be due to the weaker effect of general moxibustion. Therefore, we choose thunder-fire moxibustion as our intervention, for which the penetration is stronger than the general moxibustion.

After the burning of thunder-fire moxibustion, energy, thermal infrared radiation, medicine chemical factor, and physics factor are produced, so as to adjust human body's enginery to treat disease.. Using heat radiation, thunder-fire moxibustion improves blood circulation by the heat penetrating through the tissue [9], demonstrated by a strong effect on blood stasis and swelling as well as pain relief [24]. Compared with ordinary moxa sticks, the advantages of thunder-fire moxibustion are larger in coverage, safer, simpler operation, have higher target-point positioning accuracy, are easier to control, have lower scald risk, and reduced smog moxibustion. Moreover, thunder-fire moxibustion can alleviate other symptoms of CSR such as numbness and dysfunction.

In the previous four trials [10-13], none of them used thunder-fire moxibustion alone as the study group, so that they cannot clearly demonstrate the effectiveness of thunder-fire moxibustion. Herein, we separate the thunder-fire moxibustion or ibuprofen comparison for the sake of evaluating the practical effect of thunderfire moxibustion. Four trials [10-13] all randomly selected local lesion such as BL10 (Tianzhu), BL11 (Dazhu), EXHN15 (Jingbailao), GB20 (Fengchi), GV14 (Dazhui), GV15 (Yamen), GV16 (Fengfu), and ashi points (an acupuncture point with no specific name or definite location; the site of which is determined by tenderness or other pathological responses, also known as the ouch point). As a matter of fact, four trials were not in conformity with the acupoints selection principle of thunder-fire moxibustion. According to special moxibustion box tactics, we chose BL10 (Tianzhu), BL11 (Dazhu), and extraordinary point EX-HN15 (Jingbailao) in this study.

There are limitations to this study. One limitation is the blinding. Blinding cannot be conducted in this trial, so an open-label study is adopted, which may lead to performance bias. However, outcome measurers and statisticians will be blinded to try our best to adjust bias. Secondly, the participants will only be recruited at the 
First Affiliated Hospital of Guangzhou University of Chinese Medicine, and then the result of this trial may be suitable for Chinese people only.

The trial duration is short (a 2-week treatment), because we only focus on the short-term effect of thunder-fire moxibustion. Moreover, ibuprofen is not typically suitable for long-term use [25].

\section{Trial Status}

This protocol is version 2.0. 2019-03-17. The participants will be recruited from March 1, 2019 to June 1, 2020. But no one will be enrolled until this paper is submitted.

\section{Supplementary information}

Supplementary information accompanies this paper at https://doi.org/10. 1186/s13063-019-4012-1.

Additional file 1. SPIRIT 2013 Checklist: Recommended items to address in a clinical trial protocol and related documents.

\section{Abbreviations}

AEs: Adverse events; BP: Bodily pain; CRF: Case report form; CSR: Cervical spondylotic radiculopathy; GH: General health; MH: Mental health; NDI: Neck Disability Index; NSAIDs: Nonsteroidal anti-inflammatory drugs; PF: Physical functioning; RCT: Randomized controlled trial; RE: Emotional role limitation; RP: Physical role limitation; SF: Social functioning; SF-36: Quality of Life Assessment; SF-MPQ-2: Short Form McGill Pain Questionnaire-2; TCD: Transcranial Doppler; V: Vitality

\section{Acknowledgements}

We acknowledge the physicians and staff who assisted the study for their technical support of this protocol. We especially would like to express our gratitude to Chen Xinlin and Xu Nuo, for their kindness and helpful advice with the statistical analysis.

\section{Authors' contributions}

$\mathrm{CXH}, \mathrm{MD}$, Professor carried out the design of the study, the First Affiliated Hospital of Guangzhou University of Chinese Medicine. HYX, MD, Acupuncture Doctor, drafted the manuscript, the First Affiliated Hospital of Guangzhou University of Chinese Medicine. XDH, Acupuncture Doctor, participated in the study design and helped to draft the manuscript, the First Affiliated Hospital of Guangzhou University of Chinese Medicine. ZJB, Master of acupuncture, helped to draft the manuscript, Guangzhou University of Chinese Medicine. XBH, Master of acupuncture, helped to draft the manuscript, Guangzhou University of Chinese Medicine. HRN, Master of medicine, follow the research, the Eighth Affiliated Hospital of Sun Yat-sen University. ZMX, Ph.D of biostatistics, in charge of data analysis and helped to revise the manuscript, Kyoto Prefectural University of Medicine. ZWJ, Bachelor of medicine, help to analyze data, Shenzhen Pingle Orthopaedic Hos pital. CQ, Master of acupuncture, help to perform moxibustion and data collection, Guangzhou University of Chinese Medicine. All authors read and approved the final manuscript.

\section{Funding}

Funding for this work comes from a scientific research project of the Traditional Chinese Medicine Bureau of Guangdong Province (NO. 20183002)

\section{Availability of data and materials}

The datasets analyzed during the current study are available from the corresponding author on reasonable request.

\section{Ethics approval and consent to participate}

This study had been reviewed and approved by the Ethics Committee of the First Affiliated Hospital of Guangzhou University of Chinese Medicine (ZYYECK2018-020). All participants must provide informed consent when informed of potential risks and benefits. We will protect participants' confidentiality by a specially assigned person. There is no anticipated harm or compensation for trial participation.

Participants will be asked on the consent form whether they agree to use their data if they choose to quit the trial. Participating universities or regulators, if relevant, will also require participants to allow the research team to share relevant data with participating universities or regulators. This test does not involve the collection of biological specimens for storage. In some cases, it may be necessary to amend the protocol for the sake of clinical trial science and subject protection. We will notify the sponsor and funder first and then the PI will notify the center and a copy of the revised protocol will be sent to the PI to add to the Investigator Site File. We will also update the protocol in the clinical trial registry, and any deviations from the protocol will be fully documented using a breach report form.

\section{Consent for publication}

All participants have agreed to publish the report individually.

\section{Competing interests}

The authors declare that they have no competing interests.

\section{Author details}

${ }^{1}$ The First Affiliated Hospital of Guangzhou University of Chinese Medicine, No.12 Ji Chang Road, Baiyun District, Guangzhou 510405, China. 'Guangzhou University of Chinese Medicine, No.12 Ji Chang Road, Baiyun District, Guangzhou 510405, China. ${ }^{3}$ The Eighth Affiliated Hospital of Sun Yat-sen University, No. 3025 Shennan Middle Road, Futian District, Shenzhen 518033, China. ${ }^{4}$ Shenzhen Pingle Orthopaedic Hospital, No.252 Hangzi Section, Pingshan Avenue, Pingshan District, Shenzhen 518118, China. ${ }^{5}$ Kyoto Prefectural University of Medicine, Kajii-cho, Kawaramachi-Hirokoji, Kamigyo-ku, Kyoto 602-8566, Japan.

Received: 2 June 2019 Accepted: 19 December 2019

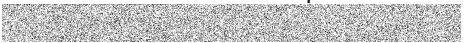

\section{References}

1. Caridi JM, Pumberger M, Hughes AP. Cervical radiculopathy: a review. HSS J. 2011;7(3):265-72.

2. Schoenfeld AJ, George AA, Bader JO, Caram PM Jr. Incidence and epidemiology of cervical radiculopathy in the United States military: 2000 to 2009. J Spinal Disord Tech. 2000;25(1):17-22.

3. GBD 2013 Disease and Injury Incidence and Prevalence Collaborators. Global, regional, and national incidence, prevalence, and years lived with disability for 301 acute and chronic diseases and injuries in 188 countries, 1990-2013: a systematic analysis for the Global Burden of Disease Study 2013. Lancet. 2015:386:743-800

4. Tanaka Y, Kokubun S, Sato T, et al. Cervical roots as origin of pain in the neck or scapular regions. Spine. 2006:31(17):568-73.

5. Tetreault LA, Rhee J, Prather $\mathrm{H}$, et al. Change in Function, Pain, and Quality of Life Following Structured Nonoperative Treatment in Patients With Degenerative Cervical Myelopathy: A Systematic Review. Glob Spine J. 2017; 7 (3 Suppl):42S-52S.

6. Rhee J, Tetreault LA, Chapman JR, et al. Nonoperative Versus Operative Management for the Treatment Degenerative Cervical Myelopathy: An Updated Systematic Review. Glob Spine J. 2017:7(3 Suppl):35S-41S.

7. Onda A, Kimura M. Comparisons between the efficacy of limaprostalfadex and pregabalin in cervical spondylotic radiculopathy: design of a randomized controlled trial. Fukushima J. Med. Sci. 2018;64(2):73-81.

8. Paul M. Peloso, M.K.A.R, J.C.M.D. Janet Lowcock and PLA.O Shi, Pharmacological Interventions Including Medical Injections for Neck Pain-An Overview as Part of the ICON§ Project. Open Orthop J. 2013;7:473-93.

9. Chen B, et al. Acupotomy versus acupuncture for cervical spondylotic radiculopathy: protocol of a systematic review and meta-analysis. BM Open. 2019;9(8):e029052 [PMID: 31399459].

10. Peng W. Clinical Controlled Trial on TongyangLishi Formula Combined with Thunder-fire Moxibustion in Treating Acute Cervical Spondylotic Radiculopathy. Chinese J Trad Feb Traum Orthop. 2014;22(5):26-30.

11. LuWei L, ChunSheng W. Observation on the Curative Effect of Thunderfire Moxibustion Combined with Traction in the Treatment of Cervical Spondylosis of Nerve Root and Vertebral Artery Type. CJGMCM. 2017; 32(17):2531-3. 
12. HuaJun Z, TingTing L, HaiDong X, Qian J, PenFa L. Research on Treating Nerve-root Type Cervical Spondylosis with Acupuncture and Moxibustion and Chinese medicine. World Chin Med. 2016;11(11):2415-8.

13. XiuYun Z, WenLi Y. Observation on the Curative Effect of Thunder-fire Moxibustion in the Treatment of Cervical Spondylosis and wind-cold obstruction pattern. Med Inf. 2010;23(3):638-9.

14. Tanaka Y. Criteria for the treatment of cervical radiculopathy. J Jpn Soc. 1993;67(3):552.

15. Li Y, Wu Y, Fan X, Sun Y. Clinical research of shoulder-arm pain of cervical spondylotic radiculopathy treated with acupuncturebased on the axillary nerve distribution. Zhongguo Zhen Jiu. 2016;36(2):135-8.

16. Chu YX, Cui WQ, Xu F, et al. Acupoint Embedding of Polyglactin 910 Sutures in Patients with Chronic Pain due to Cervical Spondylotic Radiculopathy: A Multicenter, Randomized, Controlled Clinical Trial. Evid Based Complement Alternat Med. 2018;2018:3465897. https:/doi.org/10.1155/2018/3465897.

17. Melzack R. The McGill pain questionnaire: major properties and scoring methods. Pain. 1975;1(3):277-99.

18. Vernon $\mathrm{H}$, Mior $\mathrm{S}$. The Neck Disability Index: a study of reliability and validity. J Manip Physiol Ther. 1991;14(7):409-15 [PMID:1834753].

19. Vernon H. The Neck Disability Index: state-of-the-art, 1991-2008. J Manipulative Physiol Ther. 2008;31 (7):491-502. https://doi.org/10.1016/j.jmpt.2008.08.006.

20. Jr W, Sherbourne CD. The MOS 36-item Short-Form Health Survey (SF-36). I. Conceptual framework and item selection. Med Care. 1992;30(6):473-83.

21. Wu S, Liu Z, Siyi LI. Clinical efficacy of thunder fire moxibustion on insomnia with liver qi stagnation and yin deficiency syndrome. China Med Herald. 2019:16(9):149-53

22. Xu J, Deng H, Shen X. Safety of moxibustion: a systematic review of case reports. Evid Based Complement Alternat Med. 2014;2014:783704. https:// doi.org/10.1155/2014/783704.

23. Kim SY, Chae Y, Lee SM, Lee H, Park HJ. The effectiveness of moxibustion: an overview during 10 years. Evid Based Complement Alternat Med. 2011; 2011:306515. https://doi.org/10.1093/ecam/nep163.

24. Xu D, Xu H, Liu J. Effect of Thunder-Fire Moxibustion on Pain, Quality of Life, and Tension of Multifidus in Patients with Primary Osteoporosis: A Randomized Controlled Trial. Med Sci Monit. 2018;24:2937-45.

25. Giustino V, Pergolizzi JV, Dowling P, Paladini A. Ibuprofen Safety at the Golden Anniversary: Are all NSAIDs the Same? A Narrative Review. Advances in Therapy. 2019. [PMID:31705437]. https://doi.org/10.1007/s12325-019$01144-9$.

\section{Publisher's Note}

Springer Nature remains neutral with regard to jurisdictional claims in published maps and institutional affiliations.

Ready to submit your research? Choose BMC and benefit from:

- fast, convenient online submission

- thorough peer review by experienced researchers in your field

- rapid publication on acceptance

- support for research data, including large and complex data types

- gold Open Access which fosters wider collaboration and increased citations

- maximum visibility for your research: over $100 \mathrm{M}$ website views per year

At $\mathrm{BMC}$, research is always in progress.

Learn more biomedcentral.com/submissions 